\title{
Signal-directed behavior in the rat: Interactions between the nature of the CS and the nature of the UCS
}

\author{
GRAHAM C. L. DAVEY, JOHN H. PHILLIPS, and SIMON WITTY \\ The City University, London, England
}

\begin{abstract}
Two experiments are described, which involved the investigation of interactions between the nature of the conditioned stimulus (CS) and the nature of the unconditioned stimulus (UCS) in producing signal-centered behavior. In Experiment 1, rats received response-independent heat reinforcement in a cold environment. For some groups, this heat UCS was signaled by presentations of a standard aluminum retractable lever; for other groups, it was signaled by a retractable lever covered in acrylic fur (furry lever CS). Only the subjects that received the furry lever CS paired with heat exhibited differential CS-contact behavior, when compared with unpaired, aluminum lever, and warm control subjects. In Experiment 2, hungry rats received pairings of either an aluminum or a furry lever with food (UCS). When compared with unpaired controls, only the subjects that received the aluminum lever paired with food showed differential signal-directed behavior; the subjects receiving the furry lever CS did not show differential contact with the CS, but instead exhibited differential food tray entry behavior during CS presentation. In the two studies, the signal-directed behavior exhibited by subjects resembled either thermoregulatory or feeding behaviors characteristic of rats. The results suggest that signal-directed behavior is determined by a complex interaction between the ecological relevance of the CS and the nature of the UCS-an interaction that can best be described in terms of a behavior systems model of conditioned responding.
\end{abstract}

In autoshaping studies, a localizable conditioned stimulus (CS) is programmed to precede a response-independent appetitive reinforcer (an unconditioned stimulus; hereafter UCS) such as food. In such studies, animals normally approach and contact the CS during its presentation (e.g, Brown \& Jenkins, 1968; Schwartz \& Gamzu, 1977), and CS contact behaviors frequently resemble the animal's normal UCS consummatory response (this behavior is frequently known as signal-directed or signal-centered behavior). For instance, pigeons will direct "drinking-like" pecks at an illuminated keylight CS predicting a water UCS, and "eating-like" pecks at an illuminated keylight CS predicting a food UCS (Jenkins \& Moore, 1973). Rats will bite and mouth a retractable lever CS predicting a solid food pellet UCS, but lick and sniff the same type of CS when it predicts a liquid food UCS (Davey \& Cleland, 1982). Results such as these are generally taken to be consistent with a stimulus substitution account of autoshaping which implies that the CS becomes a "substitute" or "surrogate" UCS (cf. Pavlov, 1927).

However, the results of a number of autoshaping studies do not support predictions from stimulus substitution theory, but rather suggest that signal-centered behavior is affected in a rather complex way by the nature of both the CS and the UCS (cf. Davey, 1989, chapter 6; Holland,

Correspondence may be addressed to Graham Davey, Psychology Division, The City University, Northampton Square, London ECIV OHB, England.
1984). When the nature of the UCS has been manipulated, studies have shown that some UCSs will generate very little CS-directed behavior, while other UCSs generate signal-directed behavior unlike any of the consummatory behaviors maintained by that UCS. For instance, Davey and Cleland (1982) found that when water and food were compared as UCSs paired with a retractable lever CS for rats, food maintained high levels of CS-contact behavior, whereas water generated conditioned behavior that was mainly directed at the UCS site and not at the CS. Furthermore, in an autoshaping study in which the effect of a thermal UCS on signal-directed behavior in chicks was examined, Wasserman, Hunter, Gutowski, and Bader (1975) found that presenting chicks with a 4-sec burst of radiant heat from a $250-W$ heat lamp in a cold environment produced pecking of a small keylight CS that was illuminated for 8-sec immediately prior to heat reinforcement. The conditioned response (CR) observed in this study consisted of CS-directed pecking accompanied by snuggling up to the keylight (cf. Wasserman, 1973). This kind of signal-directed response was quite unlike the chicks' response to the heat reinforcer, which was described as "basking"- -that is, the chicks "stopped scurrying about, extended their wings, and often emitted twittering sounds" (Wasserman, 1973, p. 876). These studies suggest that appetitive UCSs do not invariably generate signal-directed responding to localizable CSs, nor, when they do, does this responding necessarily resemble any component of the unconditioned response (UCR). 
Similarly, autoshaping studies in which the nature of the CS has been manipulated have also produced results that appear to be inconsistent with a stimulus substitution account. First, Timberlake and Grant (1975) paired periodic presentations of a restrained conspecific with food and found that, although the subject rats did exhibit signalcentered behavior, this behavior resembled social rather than food-consuming activities. Second, Cleland and Davey (1983) compared the effects of localizable auditory and visual CSs paired with a food UCS in rats. Although rats readily approached and contacted the visual $\mathrm{CS}$, they rarely did so in response to the auditory CSeven though they could discriminate and localize it. These results were contrary to those from a similar study by Grastyan and Vereczkei (1974), using cats as subjects. In this latter study, strong and persistent approach behavior to a localizable auditory CS in cats was found.

The general conclusions from studies such as those reported above are that (1) signal-directed behavior is not an invariable consequence of pairing a localizable CS with an appetitive UCS, and (2) when signal-directed behavior is generated, CS-contact behaviors do not necessarily resemble any component behavior of the UCR to the UCS. Clearly, any performance theory of signal-directed behavior has to address not just the nature of the UCS, but also the nature of the CS, the motivational system concerned, and the species being conditioned. One performance model of Pavlovian conditioning in which this has been attempted is the behavior systems approach to conditioned responding (Davey, 1989; Holland, 1984; Timberlake, 1983). This approach reflects an attempt to relate three specific aspects of an animal's behavior: phylogenetically preorganized responses, internal motivational states (such as a deprivation state like hunger), and external stimuli that may act to release responses relevant to that motivational state. This account also makes some specific predictions about the kind of CRs that are likely to occur in an appetitive autoshaping experiment. First, signal-directed CRs are only likely to be observed if features of the CS resemble the natural cues for releasing autotaxic responses in that behavior system (i.e., the stimulus possesses features that the animal will tend to approach and investigate when in a relevant motivational state). For instance, rats normally approach and investigate small, manipulable objects when feeding; the retractable levers used as appetitive CSs in many autoshaping studies are also small, manipulable objects, which, prima facie, possess features appropriate for releasing this investigative foraging activity (cf. Barnett, 1956; Davey, 1989; Davey \& Cleland, 1984). Rats also approach and contact a conspecific CS for food, and this appears to be related to the fact that rats are social feeders that tend to approach other rats when feeding (Timberlake, 1983; Timberlake \& Grant, 1975). However, rats do not approach a localizable auditory CS paired with food; and, consistent with this finding is the fact that they do not locate food on the basis of auditory cues. Thus, this type of stimulus is ineffective in releasing components of the feeding behavior system (Cleland \& Davey, 1983; Harrison, 1979). Nevertheless, auditory CSs are effective for other species. Cats do autoshape to a localizable auditory CS, and this species consists of carnivorous predators that often locate their prey on the basis of auditory signals. Thus, for feeding cats, sounds do possess an ecological relevance, and they act to release approach and investigative responses (Grastyan \& Vereczkei, 1974).

The present study comprises two experiments designed to test further predictions from the behavior systems model of Pavlovian performance. The first experiment is an investigation of the nature of the CRs generated by a thermal UCS in rats, using both ecologically relevant and irrelevant CSs. In the second experiment, the same CSs as in Experiment 1 were used, but they were paired with an alternative UCS, food.

\section{EXPERIMENT 1}

In a thermal conditioning study, Wasserman et al. (1975) found that a 4-sec burst of radiant heat from a 250-W heat lamp in a cold environment could act as an effective UCS in generating signal-directed responding in chicks. Chicks quickly learned to approach and peck at an 8-sec keylight illumination, which was paired with the heat burst. However, preliminary thermal conditioning studies with rats carried out in our laboratory have failed to demonstrate any sustained signal-directed behavior when heat is paired with presentations of a conventional retractable lever CS (Griffiths \& Davey, 1984). This latter failure is in strict contrast to the substantial amount of signal-directed behavior that is evoked by a retractable lever CS when the UCS is food (e.g., see Davey \& Cleland, 1982; Davey, Oakley, \& Cleland, 1981). One explanation for this anomaly may lie in the ecological relevance of the cues possessed by a retractable lever CS when heat or food is used as the UCS. The small, manipulable nature of the lever CS has a relevance to feeding, since rats regularly approach and investigate small edible and inedible objects when feeding (Barnett, 1956). However, there is no reason to suppose that a rat that is seeking warmth should approach and investigate a small metal object such as a retractable lever. So what cues might a localizable CS need to possess in order to generate signal-directed responding using a thermal UCS?

One important behavioral method of thermoregulation in the rat is social huddling or clumping. This behavior, found in newly born rat pups, continues into adult life as an important means of thermoregulation and energy conservation (cf. Alberts, 1978a; Barnett, 1963; Calhoun, 1962). A rat huddle is an active, dynamic mass with members continually reorienting and adjusting within the huddle and toward the huddle if they become detached from it (Alberts, 1978a). Alberts (1978b) has investigated some of the sensory cues that elicit and maintain huddling in rat pups. These include immobile conspecifics, olfactory cues, and furry "comfort" cues. Such cues all elicit autotaxic responses and eventual huddling. Experiment 1 was 
designed to compare the effects of both ecologically relevant and ecologically irrelevant CSs (a "furry" lever and an aluminum lever, respectively) on signal-directed behavior, using a thermal UCS.

\section{Method}

\section{Subjects}

The subjects were 24 Hooded Lister rats approximately 120 days old at the outset of the experiment. They were all housed in pairs with ad-lib food and water available at all times. All subjects were experimentally naive at the outset of the experiment, and they were held in an animal holding room with an ambient temperature of $20^{\circ} \pm 2^{\circ} \mathrm{C}$.

\section{Apparatus}

The experimental environment consisted of a specially built aluminum chamber, whose dimensions were $41 \times 33 \times 30 \mathrm{~cm}$. On the longest wall of this chamber was mounted an aluminum retractable lever $7 \mathrm{~cm}$ wide and $4 \mathrm{~cm}$ thick, which reached $3.5 \mathrm{~cm}$ into the chamber when extended. When retracted, the lever was flush with the wall of the chamber. It took approximately $0.5 \mathrm{sec}$ to extend the lever fully into the chamber. This lever was situated $6 \mathrm{~cm}$ from the right-hand wall and $2.5 \mathrm{~cm}$ above the grid floor. In some experimental conditions (see below), all protruding surfaces of the lever were covered with acrylic fur (this was called the furry lever). The fur, which was glued to the surfaces of the lever, consisted of patches of black and white similar to the markings found on Hooded Lister rats.

Situated centrally above the chamber approximately $23 \mathrm{~cm}$ from the grid floor was a 250-W infrared heat lamp, which could be activated for 4-sec periods to provide radiant heat reinforcement. Situated approximately $50 \mathrm{~cm}$ above the grid floor of the chamber was a closed-circuit TV camera, which, with the use of a wide-angled lens, could relay TV pictures of the subject to observers in an adjoining room. The experimental chamber and TV camera were all housed in a cooled incubator where the ambient temperature controls were set at $0^{\circ} \pm 2^{\circ} \mathrm{C}$. General illumination in the cooled incubator was provided by a $15-\mathrm{W}$ lamp situated $20 \mathrm{~cm}$ directly above the heat lamp. The experiment was controlled and data collected by a specially designed on-line microcomputer system.

\section{Procedure}

The subjects were divided into six groups of 4 subjects each. These groups were labelled FC+, FC-, LC+, LC-, $\mathrm{FW}_{+}$, and FW-, respectively.

Group FC+ (furry lever/cold box/paired). This group received 8 sessions of autoshaping training, in which 4-sec activations of the heat lamp (UCS) were immediately preceded by 10 -sec presentations of the furry lever (CS). Pairings of the CS and UCS were scheduled on a variable-time 60 -sec schedule with a minimum intertrial interval of $30 \mathrm{sec}$ and a maximum of $90 \mathrm{sec}$. The subjects received $\mathbf{4 0}$ trials per session, and the experimental chamber was maintained at an ambient temperature of $0^{\circ} \pm 2^{\circ} \mathrm{C}$.

Group FC- (furry lever/cold box/unpaired). This group received 8 sessions in which the furry lever (CS) and heat lamp presentations (UCS) were scheduled on identical but independent variabletime 60-sec schedules. The heat lamp and furry lever could not be presented simultaneously; if either stimulus was being presented when the other was scheduled, the scheduled stimulus was held over until 5-sec after the offset of the presented stimulus. The subjects received 40 presentations of both the heat lamp and the furry lever during a session, and the experimental chamber was maintained at $0^{\circ} \pm 2^{\circ}$ throughout.

Group LC+ (aluminum lever/cold box/paired). These subjects received exactly the same training as did Group FC+, except that the retractable furry lever was replaced from the outset with an identically sized retractable aluminum lever.
Group LC - (aluminum lever/cold box/unpaired). These subjects roceived training identical to that of the subjects in Group FC-, except that a retractable aluminum lever replaced the retractable furry lever.

Group FW+ (furry lever/warm box/paired). This group received pairings of the furry lever $\mathrm{CS}$ and heat reinforcement identical to that received by Group FC +, except that the experimental chamber was maintained at an ambient temperature of $20^{\circ} \pm 2^{\circ} \mathrm{C}$. This group was included to assess the effectiveness of the heat lamp reinforcer in warm versus cold environments.

Group FW- (furry lever/warm box/unpaired). This group received training identical to that of Group FC-, except that the ambient temperature in the experimental chamber was maintained throughout training at $20^{\circ} \pm 2^{\circ} \mathrm{C}$.

\section{Observational Procedures}

For all groups of subjects, Sessions 1 and 8 of training were videorecorded, and the behaviors of each subject were analyzed according to preselected topographical categories that were defined on the basis of casual observations made during pilot studies. Each category was scored on the basis of the percentage of trials on which at least one instance of the behavior occurred. The behavioral categories used were of two basic types: CS-directed responses, and nondirected responses. CS-directed categories included (1) $C S$ contact-contact between the CS and any part of the subject's body; (2) orient-standing motionless on all four legs and turning the head rapidly in the direction of the CS; (3) sniffing-moving the nose within approximately $1 \mathrm{~cm}$ of the CS with movements of the vibrissae characteristic of sniffing an object; (4) body contact-contact with the CS by any part of the subject's body other than the head or paws; (5) pawing - contacting the lever with one or two paws; and (6) head under-pushing the head under the lever, a response very similar to the rooting behavior found when a rat is entering a huddle. Categories of non-CS-directed behavior included (1) huddle-remaining still in a hunched position for at least $4 \mathrm{sec}$; (2) groom-grooming the head or body with either mouth or paws; (3) walk-the chamber was arbitrarily divided into six equal sections, and walking was defined as moving from one of these sections into at least one other section; (4) rear-rising up on hind legs with forepaws off the ground; and (5) rear and sniff-rising up on the hind legs with snout raised and movement of the vibrissae characteristic of sniffing an object or scenting the air. Two observers (one trained and experienced in observation procedures, and one untrained) independently scored the behaviors from two randomly selected sessions with $78.3 \%$ agreement on all categories across all trials observed. The scores given in the results represent those from the single trained observer.

These categories were used not only to analyze the behaviors that occurred during CS presentation, but also to assess the behaviors that were differentially emitted during presentation of the heat lamp UCS. Behaviors were recorded that occurred during the 4-sec heat lamp presentations and in an equal number of arbitrarily defined 4-sec periods during the intertrial intervals (designated no-UCS or UCS periods). This analysis was intended to discover if any behaviors differentially elicited by the UCS were also emitted during CS presentations.

\section{Results and Discussion}

Figure 1 shows CS-contact rates for the first and last session of training for all groups of rats. This measure exhibited a significant group $\times$ session interaction $[F(5,16)=2.97, p<.05]$, in which there was no significant difference between groups in Session $1(F<1)$, but a significant difference between groups in Session 8 $[F(5,18)=3.11, p<.05]$. A Duncan's multiple range test revealed that in Session 8, Group FC+ contacted the 


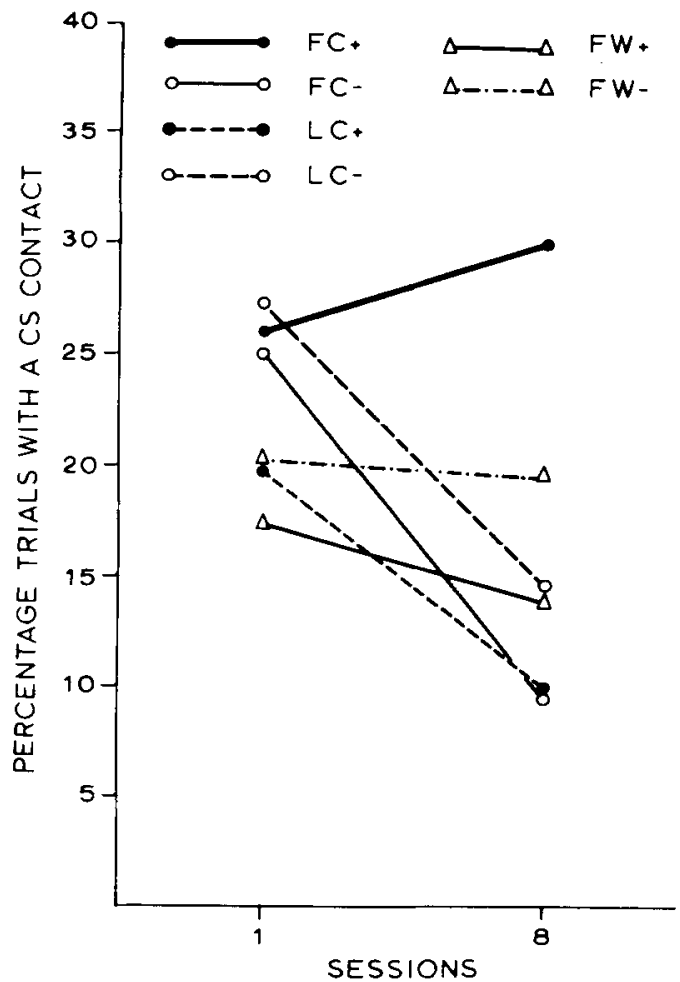

Figure 1. Percentage trials with a CS contact on Sessions 1 and 8 for all six groups in Experiment 1.

CS on more trials than did Groups $\mathrm{FC}-, \mathrm{LC}+, \mathrm{LC}-$, and FW+ (all $p s<.05$ ). There were no other significant differences.

Figure 2 presents the percentage trials on which different CS-directed behaviors occurred in the last session of training (Session 8 ). For the CS-orienting response there was a significant difference between groups $[F(2,18)=$ $5.70, p<.05]$. Duncan's test revealed that Group FC+ exhibited more CS-directed orienting than all other groups, and Group FW+ oriented significantly more than Groups FW-, LC+, and LC- . Furthermore, while there was no significant difference between groups in the level of CS sniffing $[F(2,18)=1.19, p>.1]$, there was a significant difference between groups in the frequency of paw contact with the CS $[F(2,18)=4.38, p<.05]$. Group FC+ exhibited significantly more paw contact with the CS than did all other groups (Duncan's test, all $p \mathrm{~s}<.01$ ); there were no other significant differences between groups on this measure. There was also a significant difference between groups in body contact with the $\operatorname{CS}[F(2,18)=3.83, p<.05]$, with Group FC+ showing significantly more body contact responses than all other groups except Group FW- (Duncan's test, all ps < .05). Finally, although Group FC+ appeared to exhibit more head-under responses than any other group, differences between groups on this measure failed to reach significance at the $5 \%$ level $[F(2,18)=2.97, p<.055]$.

There were no significant differences between groups in any of the other topographical categories (all $F \mathrm{~s}<1$ ).
These included rear, rear and sniff, walk, huddle, and groom.

Figure 3 shows the results of the analyses of behaviors occurring during the $4 \mathrm{sec}$ of UCS presentation and corresponding $4 \mathrm{sec}$ of no-UCS ( $\overline{\mathrm{UCS}}$ ) periods. Only rear and sniff occurred in significantly different proportions of UCS and no-UCS periods $[F(1,44)=6.46, p<.025]$. Both the cold box groups ( $\mathrm{FC}+, \mathrm{FC}-, \mathrm{LC}+, \mathrm{LC}-)$ and the warm box groups (FW+, FW-) showed significantly more rear and sniff during UCS periods than during nonUCS periods (Duncan's test, $p<.01$ and $p<.05$, respectively). Both huddle and walk did show an effect of temperature, with cold box subjects huddling more frequently than warm box subjects, and warm box subjects walking more frequently than cold box subjects during both UCS and non-UCS presentations (in all cases $F>4$, $p<.05$ ). There was no significant effect of either temperature or UCS/no-UCS on rearing and grooming.

These results indicate that rat subjects that have a furry lever CS paired with heat reinforcement in a cold environment (1) maintain levels of contact with the CS that are significantly higher than those shown by unpaired, alu-

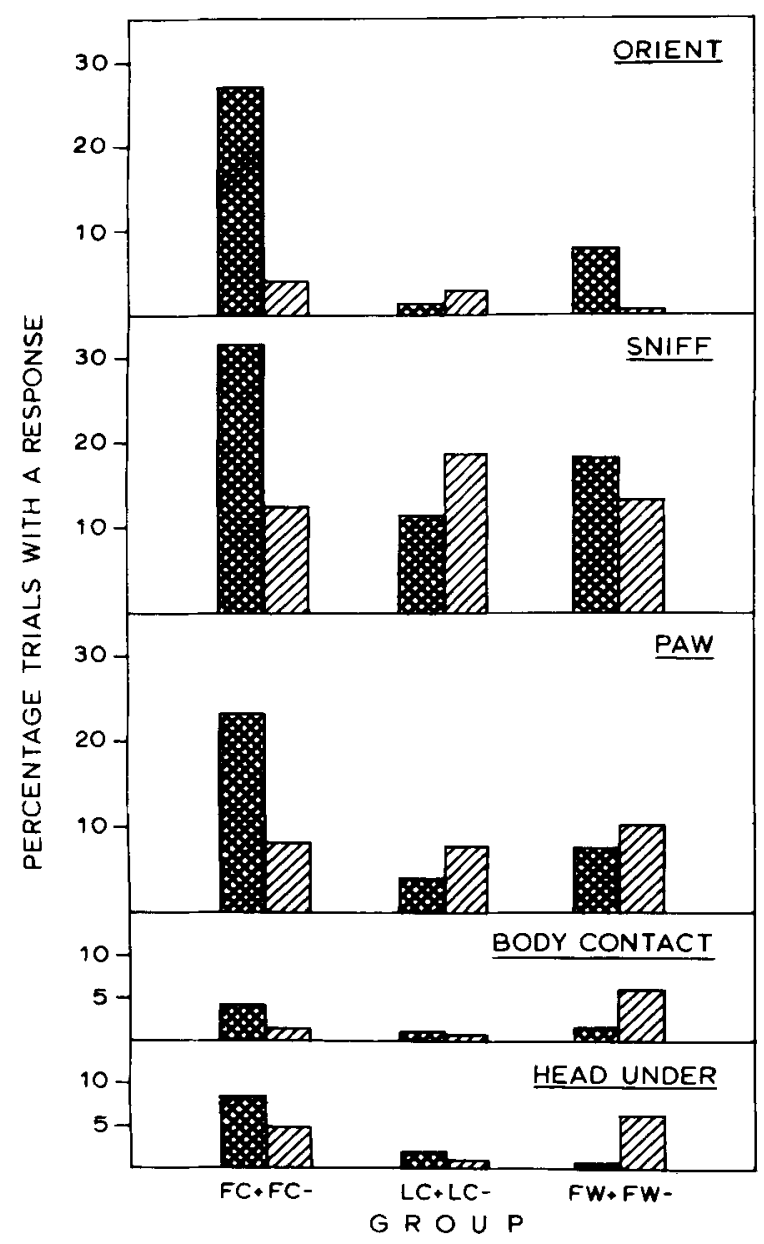

Figure 2. Percentage trials with an orient, sniff, paw, body contact, or head-under response for all six groups on Sescion 8 of Experiment 1 . 


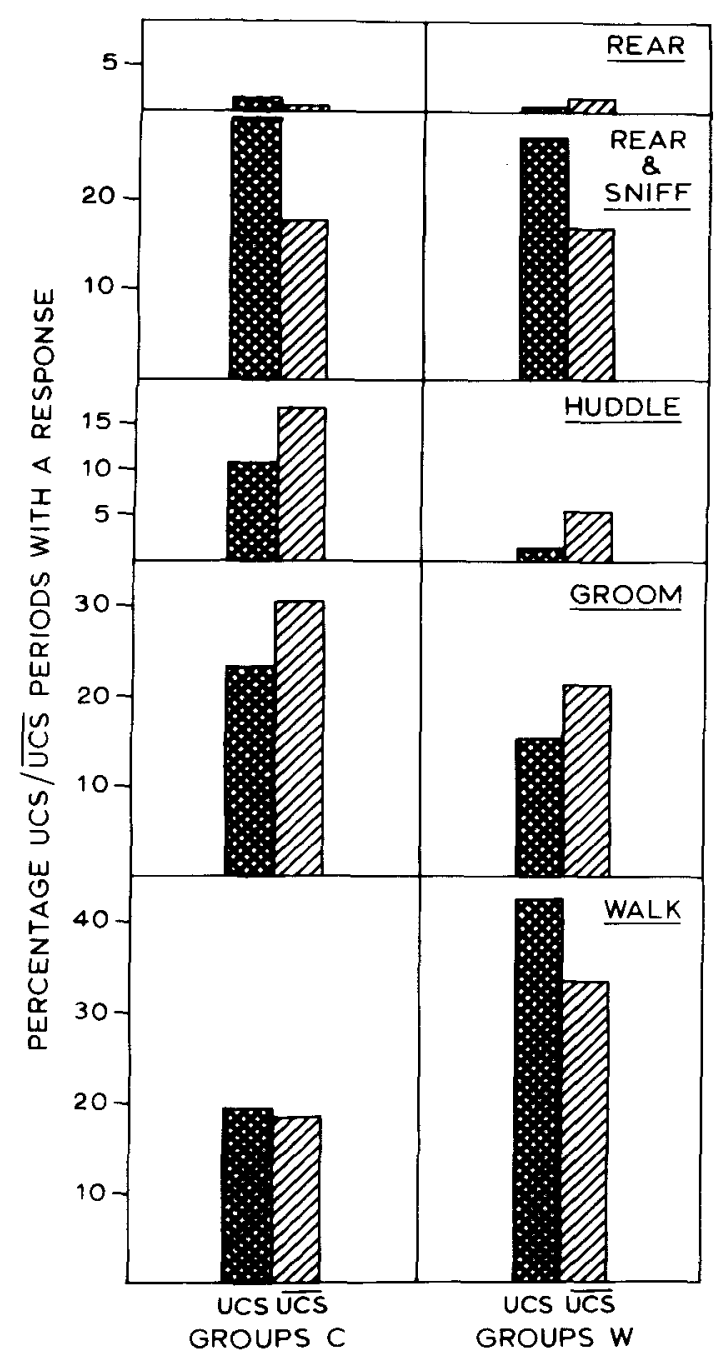

Figure 3. The percentage UCS presentations and equivalent noUCS periods containing a response for cold box subjects (Groups C) and warm box subjects (Groups W) from Session 8 of Experiment 1. Hatched bars represent behaviors occurring during the UCS (heat presentation); open bars represent behaviors occurring during an equivalent 4-sec period during the intertrial interval.

minum lever, and warm box control subjects, (2) exhibit higher levels of orienting to and pawing of the CS than do appropriate unpaired, aluminum lever, and warm box controls, and (3) show significantly higher levels of body contact with the CS than do unpaired and aluminum lever control subjects (although the absolute levels of this response were low in all groups). The only differential response to the heat UCS that was recorded consisted of rearing and sniffing, found in both cold box and warm box subjects. However, neither rearing and sniffing nor sniffing at the CS became differential CRs occurring during CS presentation. The only differential CRs recorded were CS-directed responses. This latter finding is clearly inconsistent with predictions from stimulus substitution accounts of autoshaping, since there was no obvious evi- dence of the transfer of UCRs to the CS; certainly, the differential pawing observed in Group FC+ was a response that was never observed as being directed toward the heat lamp UCS.

One possible explanation for the differential signaldirected behavior observed in Group FC+ can also be ruled out. It might have been possible that initial orienting reactions could have directed the animal toward the CS, and that approach and contact could then have been reinforced by the comfort derived from contact with the furry lever. However, if this were the case, one might also expect subjects that had unpaired CS presentations to adopt a similar strategy. Presumably, if the furry lever had any comfort value beyond its association with the UCS (such as a marginal amount of warmth), this would be manifested in both paired and unpaired groups. Since levels of CS contact were clearly different in paired and unpaired groups, then what maintains CS-contact in the paired group must to some extent derive from some aspect of the CS-UCS relationship.

That differential signal-directed responding was maintained by the furry CS and not by the aluminum CS is further evidence that the nature of the CS is important in determining signal-directed behavior. Furthermore, these findings are consistent with the prediction, made on the basis of a behavior systems account of performance, that autotaxic responding should only be observed when the CS possesses some ecological relevance to the UCS and the motivational system concerned. However, it may simply be the case that a furry lever CS generates differential signal-directed responding regardless of the nature of the UCS-either because it possesses extra salience and thus establishes stronger CS-UCS associations, or because, for the species concerned, it has some kind of nonspecific relevance that generates autotaxic behaviors in response to pairing with any biologically significant event. In the second experiment, we investigated some of these possibilities by comparing the autotaxic properties of furry and aluminum levers paired with a different UCS, namely food.

\section{EXPERIMENT 2}

When a retractable lever CS is paired with a food UCS for rat subjects, vigorous and sustained CS-directed behavior is usually observed (e.g., see Davey \& Cleland, 1982; Davey, Oakley, \& Cleland, 1981; Peterson, Ackil, Frommer, \& Hearst, 1972), and the nature of the CS-directed behavior frequently resembles the nature of the consummatory response to the food UCS (e.g., see Davey \& Cleland, 1982). In behavior systems terms, this is explained through allusion to the fact that the lever is a small manipulable object that releases approach and investigative behaviors in a feeding rat (cf. Davey, 1989; Davey \& Cleland, 1984). Thus, a behavior systems account would predict significant differential responding to an aluminum lever CS paired with food compared with the lack of differential responding to one paired with heat (Experi- 
ment 1). To the extent that a furry lever resembles a conspecific rat, this stimulus may also possess some ecological relevance when paired with food. Timberlake and Grant (1975) found signal-directed responding in rats to a restrained conspecific paired with food. The nature of this responding, however, did not resemble the consummatory behavior to the food UCS but consisted of social behaviors such as anogenital sniffing and mouth sniffing. Timberlake and Grant (1975) and Timberlake (1983) have attempted to explain these findings in behavior systems terms by alluding to the fact that rats are social feeders, so that, as a releasing stimulus for autotaxic behavior, a conspecific CS has some ecological relevance. Thus, if superficial external resemblance to a conspecific is the important cue in releasing social feeding behaviors, we might expect that a furry lever paired with food would generate some differential CS-directed behavior. Nevertheless, on this basis we would still expect the actual nature of CS-centered behavior to differ between aluminum and furry levers in that the CRs to the aluminum lever should be primarily orally and manually investigative (e.g., biting, licking, sniffing, pawing), whereas CRs to the furry lever should be primarily social (e.g., sniffing and pawing). This second experiment is an investigation of the nature of the CRs generated by furry and aluminum lever CSs when they are paired with a food UCS, relating the findings to predictions from a behavior systems account of Pavlovian performance.

\section{Method}

\section{Subjects}

The subjects were 16 Hooded Lister rats approximately 120 days old and maintained at approximately $80 \%$ of their ad-lib body weights throughout the experiment. They were all housed in pairs, with water available continuously in their home cages.

\section{Apparatus}

The experimental chamber was a specially built Skinner box $30 \times 25 \times 18 \mathrm{~cm}$ (smaller than the chamber used in Experiment 1 ). Situated in one wall of the box was a central reinforcer-tray recess $6 \mathrm{~cm}$ high and $5 \mathrm{~cm}$ wide. The perspex flap that covered this recess could, when pushed, be used to record tray entries via a microswitch connected to the top of the flap. Reinforcement was provided in the form of a single 45-mg food pellet delivered into the food tray and accompanied by a brief flash of the tray light. Situated $2 \mathrm{~cm}$ to the left of the tray and $2 \mathrm{~cm}$ above the grid floor was a retractable lever identical to the one used in Experiment 1. This lever had the same dimensions as the one used in Experiment 1 and extended $3.5 \mathrm{~cm}$ into the chamber. When retracted, the lever was flush with the wall of the chamber. Just as in Experiment 1, in some experimental conditions this aluminum lever was covered with acrylic fur; in other conditions, it was not covered with fur and was used as a normal aluminum retractable lever. A small houselight situated on the ceiling of the chamber provided general illumination throughout each session. The chamber was housed in a sound attenuating box with the front door open to permit observation of the subjects through closed-circuit television. A closed-circuit TV camera, positioned in front of the chamber throughout the whole experiment, relayed TV pictures of the subjects to observers in an adjoining room. The experiment was controlled and data collected by a specially designed on-line microcomputer system.

\section{Procedure}

The subjects were divided into four groups of 4 subjects each. These groups were labeled F+, F-, L+, and L-, respectively. All subjects were then given identical magazine training, which consisted of four sessions of food pellets delivered on a variable-time $60-\mathrm{sec}$ schedule. At the end of this period of magazine training, all subjects were taking the food pellets soon after delivery.

Group F+ (furry lever/paired). This group received 8 sessions of autoshaping training in which food pellets (UCS) were immediately preceded by $10-\mathrm{sec}$ presentations of the furry lever (CS). Pairings of CS and UCS were scheduled on a variable-time 60-sec schedule with parameters identical to those in Experiment 1. The subjects received $\mathbf{4 0}$ trials per session.

Group F- (furry lever/unpaired). This group received 8 sessions in which the furry lever (CS) and food (UCS) were scheduled on identical, but independent, variable-time 60 -sec schedules. The only constraint on this schedule was that food could never be presented during the time that the lever was also being presented. The subjects received 40 presentations of both furry lever and food during a session.

Group L+ (aluminum lever/paired). These subjects received exactly the same training as Group F+, except that the retractable furry lever was replaced from the outset by an identically sized retractable aluminum lever.

Group L- (aluminum lever/paired). These subjects received identical training to the subjects in Group F-, except that a retractable aluminum lever replaced the retractable furry lever.

\section{Observational Procedures}

For all groups of subjects, Session 8 of training was videorecorded and the behaviors of each subject were analyzed according to preselected topographical categories. Because contacts with the furry lever could not be recorded directly, these were also determined by observation during Session 1 and compared with contact levels in Session 8. Behaviors in Session 8 were analyzed according to the topographical categories adopted in Experiment 1, with the addition of a single new category. This was biting, defined as grasping part of the lever with the teeth and making movements of the mouth similar to gnawing or biting an object. Two observers independently scored the behaviors from two randomly selected sessions, with $85.3 \%$ agreement between them. As with the observational data presented in Experiment 1, the scores given in the results represent those from the single experienced observer.

\section{Results and Discussion}

Figure 4 shows the percentage trials with a CS contact on Sessions 1 and 8 for all four groups. This exhibited a significant main effect of groups $[F(3,9)=8.55$, $p<.01$ ], with Group L+ exhibiting significantly more trials with a CS contact than all other groups (Duncan's test, all $p \mathrm{~s}<.01$ ). There were no significant differences between any of the three other groups $\left(F+, F-, L_{-}\right)$on this measure. The acquisition of a differential CR in the first session of training for Group $L+$ reflects the very rapid acquisition of signal-directed behavior previously found in rats using an aluminum lever CS and a food UCS (e.g., Davey \& Cleland, 1982; Davey, Oakley, \& Cleland, 1981).

Figure 5 shows the percentage trials with a food tray entry for all four groups in Sessions 1 and 8 of training. This measure exhibited a significant main effect of groups $[F(3,9)=19.73, p<.001]$, in which, by Session 8, Group F+ was exhibiting significantly higher levels of 


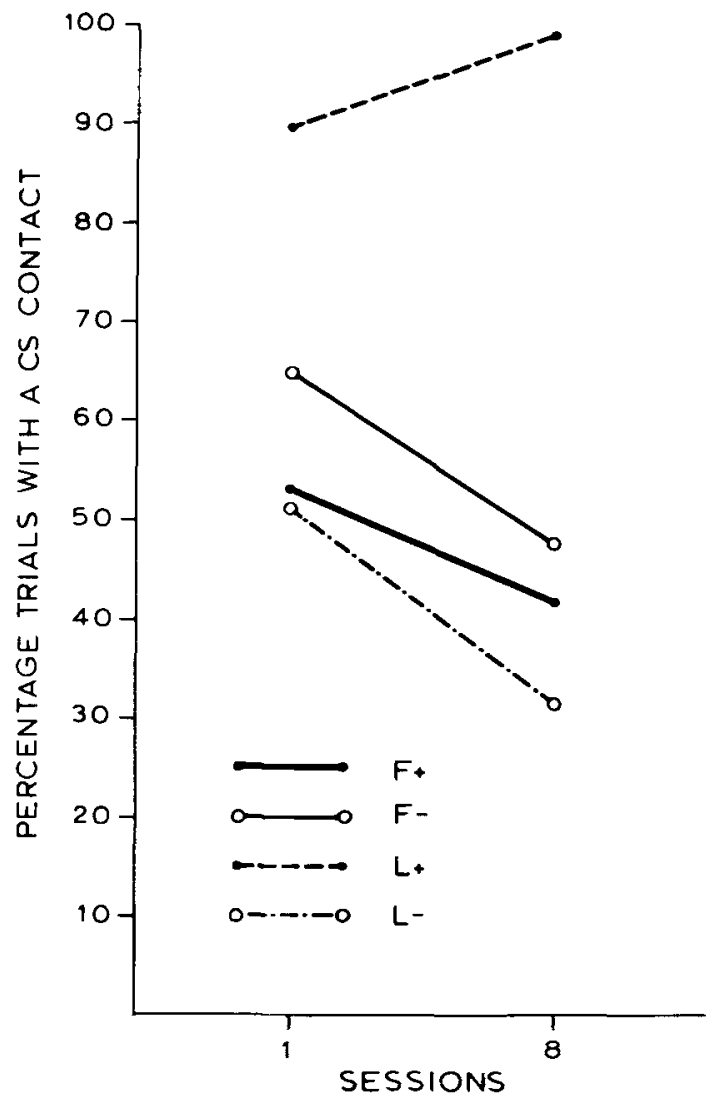

Figure 4. Percentage trials with a CS contact on Sessions 1 and 8 for all four groups in Experiment 2.

tray entry during CS presentation than all other groups (Duncan's test, all $p s<.01$ ). In Session 8, there was no difference in tray entry rate between Groups $\mathrm{F}-$ and $\mathrm{L}-$ $(p>.05)$, but both of these groups had a significantly higher rate of tray entry than did Group L+ (Duncan's test, both $p s<.05$ ).

The two main CS-directed behaviors observed in Session 8 were biting and pawing. They are presented in Figure 6. Biting showed a significant group effect $[F(3,12)=18.60, p<.0001]$ in which Group $L+$ exhibited a significantly greater level of CS biting than did all other groups (Duncan's test, all $p s<.05$ ). The only other significant difference on this measure was that Group F- exhibited more CS biting than did Group L$(p<.05)$. Pawing also exhibited a significant group effect $[F(3,12)=87.04, p<.0001]$, in which Group L+ pawed the CS on significantly more trials than did all other groups (Duncan's test, all $p s<.05$ ), and Group F+ pawed the CS on significantly fewer trials than did all other groups (Duncan's test, all $p$ s $<.05$ ). There were no significant group effects on the remaining CS-directed behaviors, which included orient, sniff, body contact, and head under (all $F \mathrm{~s}<1$ ).

Figure 7 shows the frequency of non-CS-directed behaviors occurring during CS presentation on Session 8. Walking exhibited a significant group effect $[F(3,12)=$
26.26, $p<.0001$ ], in which Group L- exhibited significantly more walking than did all other groups (Duncan's test, all $p s<.01$ ). Sniffing also showed a significant group effect $[F(3,12)=5.72, p<.05]$, in which Groups $\mathrm{L}-$ and $\mathrm{F}+$ exhibited significantly more sniffing than did Group L + . There were no significant group effects for rearing and grooming.

The main results from Experiment 2 show that (1) rats given pairings of an aluminum lever CS with food exhibit substantial amounts of CS-directed behavior when compared with appropriate controls, (2) rats given pairings of a furry lever CS with food show levels of CS-directed behavior that are no greater than those exhibited by unpaired control subjects, (3) the main differential CR shown by furry lever subjects was food tray entry, whereas (4) aluminum lever subjects showed significantly less food tray activity than did unpaired controls, and (5) the major CS-directed behaviors observed in the aluminum lever subjects were CS biting and pawing.

In strict contrast to the results of Experiment 1, this second study shows that an aluminum lever is more powerful than a furry lever in generating signal-directed behavior when food is used as the UCS. This implies that the superiority of the furry lever in Experiment 1 was not simply the result of furry levers' possessing some nonspecific saliency that either produces better conditioning

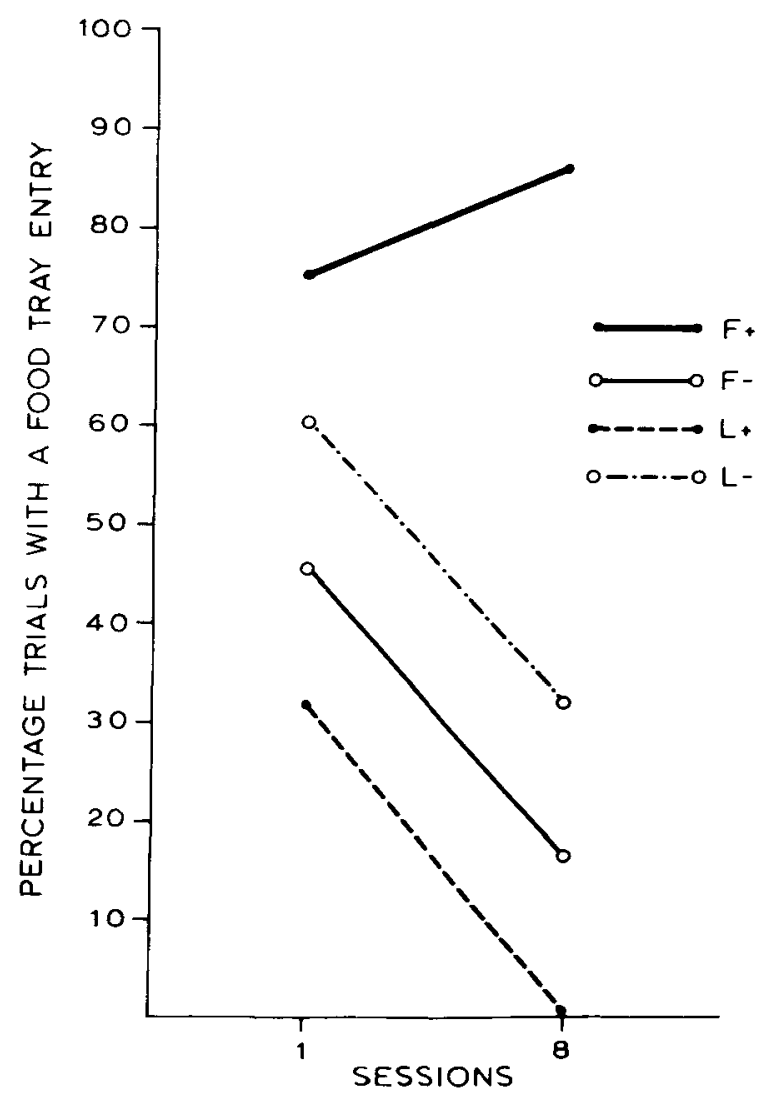

Figure 5. Percentage trials with a food tray entry on Sessions 1 and $\mathbf{8}$ for all four groups in Experiment 2. 


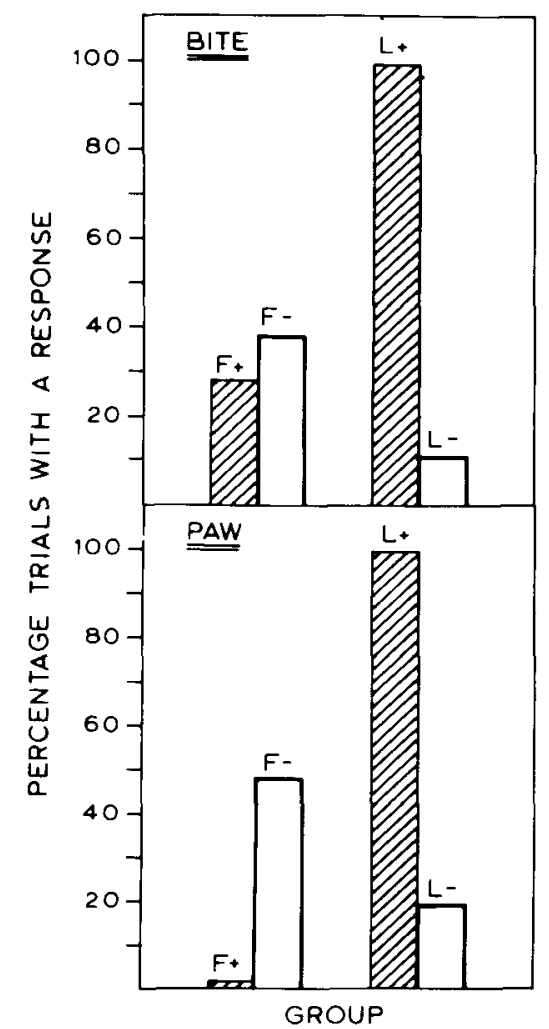

Figure 6. Percentage trials with a CS-directed bite or paw for all four groups on Session 8 of Experiment 2.

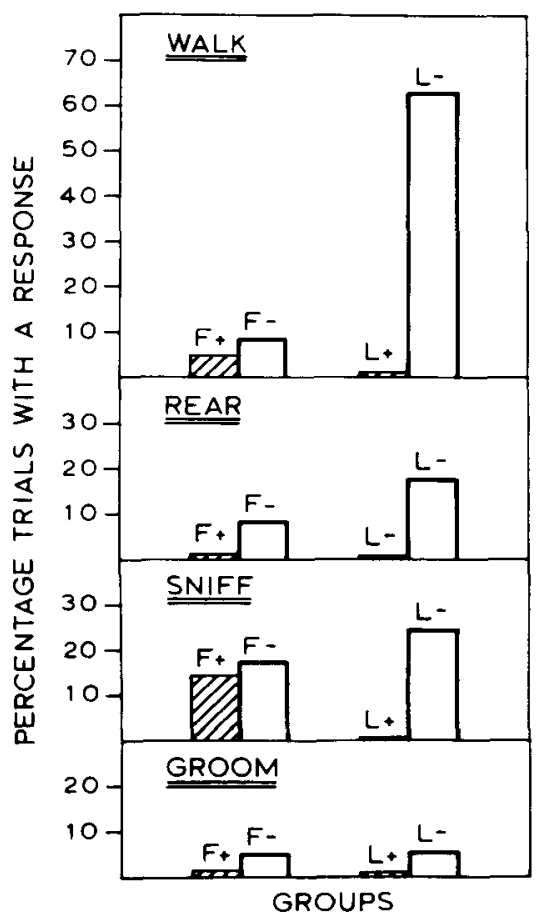

Figure 7. Percentage trials containing walking, rearing, sniffing, or grooming responses for all four groups on Session 8 of Experiment 2. or has some kind of nonspecific ecological relevance regardless of the nature of the UCS. Signal-directed behavior is clearly determined by some interaction between the nature of the CS and the nature of the UCS.

Observed behaviors in response to the aluminum lever CS (Group L+) consisted mainly of biting and pawing the lever, findings which are clearly comparable to the results of other studies involving the use of similar metal levers and a food pellet UCS (e.g., Cleland \& Davey, 1982; Davey \& Cleland, 1982; Davey, Phillips, \& Cleland, 1981). The main differential CR elicited by the furry lever CS, however, consisted of approach and entry to the food tray. In previous studies, such a CR has been observed when the CS is one that does not possess any direct ecological relevance to the UCS (e.g., Cleland \& Davey, 1983 , using a localizable auditory CS with a food UCS; Davey \& Cleland, 1982, using a water UCS with an aluminum lever (S), and hence does not generate autotaxic behavior. However, it was suggested in the introduction to this experiment that the furry lever may possess some ecological relevance to food, inasmuch as it resembles a conspecific. Clearly, the results were contrary to this prediction, and $\mathrm{F}+$ subjects did not emit signal-directed responses at a level any higher than either $\mathrm{F}-$ or $\mathrm{L}-$ control subjects. Either the features of the furry lever were insufficiently similar to a conspecific to endow it with any ecological relevance, or some other as yet unexplained reason prevented any ecological relevance from generating autotaxic behavior.

\section{GENERAL DISCUSSION}

The results of the two experiments reported in this paper suggest that signal-directed behavior is generated not by features of either the CS or UCS alone, but by an interaction between the two. A furry lever CS generated differential autotaxic responding when heat in a cold environment was the UCS, but not when food was the UCS. Similarly, an aluminum lever CS generated substantial signal-centered behavior when food was the UCS but not when heat was the UCS. These results appear to suggest that signal-directed responding is not determined solely by the salience or nonspecific biological relevance of the CS itself, but by the relevance of the CS in the context of a particular reinforcer or motivational system.

Both Timberlake (1983) and Davey (1989) have attempted to explain these CS-UCS interactions in a theory of Pavlovian performance based on a behavior systems analysis of conditioned responding. In this approach, CSs would only elicit signal-directed behavior if the CS has an ecological relevance to the UCS and acts as a natural releaser for an integrated response subsystem containing autotaxic components. This model explains why an aluminum lever CS would not generate differential signal-directed behavior when paired with a heat UCS (because it contains no obvious features that release thermoregulatory responses in rats), but does generate such 
responding with a food UCS (because it resembles the small manipulable objects that feeding rats would normally approach and investigate while feeding; cf. Barnett, 1956; Ewer, 1971). The observed effects of a furry lever CS paired with a heat UCS were clearly within the bounds of a behavior systems interpretation. First, "furriness" is one of the cues that has been shown to elicit the thermoregulatory response of huddling or clumping in cold rats (cf. Alberts, 1978b), and indeed many of the responses differentially directed toward this CS resembled the responses of rats attempting to huddle. These responses included stationary body contact with the lever, the headunder responses typical of rats attempting to join a huddle, and, most commonly, pawing of the lever-which in many cases resembled the rooting responses also typical of rats attempting to join a huddle. Second, the failure to find differential signal-directed behavior when a furry lever was paired with food initially suggests that the CS ought to possess little, if any, ecological relevance in these circumstances. However, if the furry lever possessed some cues that caused it to resemble a conspecific, then the behavior systems analysis would have predicted some signal-directed behavior on the basis that such cues should release components of the social feeding subsystem (cf. Timberlake, 1983; Timberlake \& Grant, 1975). There are at least two possible explanations for this discrepancy.

First, it may simply have been the case that although the furry lever superficially resembled a conspecific, it may not have possessed the specific cues necessary for releasing social feeding activities. A number of laboratory and field studies have shown that rats will normally approach other rats that are feeding (e.g., Ewer, 1971; Steininger, 1950; Stimber, Schaeffer, \& Grimsley, 1966), and weanling rats prefer to feed with another rat rather than alone (Galef, 1978). Nevertheless, it is not clear what conspecific cue, or combination of cues, releases this tendency, and it may be that the furry lever did not possess the relevant cues. Such cues might include conspecific odors or cues that provide evidence that a conspecific is feeding.

Second, while the furry lever CS may have possessed some ecological relevance for a feeding rat, it may not have possessed the specific physical properties necessary to support the feeding activities directed toward it. For instance, two of the most prominent behaviors directed at a conspecific CS for food are anogenital sniffing and mouth sniffing (Timberlake \& Grant, 1975), yet a furry lever has neither an anogenital region nor a mouth. As such, the furry lever has no means of supporting these behaviors. If such behaviors are essential components of an integrated chain of social feeding responses, then the rat's inability to perform them on the furry lever may have disrupted and extinguished this chain. Unfortunately, while either of these two explanations is plausible, a proper assessment awaits further knowledge of the structure and dynamic characteristics of social feeding in rats-information that is necessary for assessing the ultimate validity of a behavior systems analysis.
Finally, the results of the present study suggest that signal-centered behavior is determined by a complex interaction between CSs and UCSs, and that it is not a simple consequence of pairing a localizable CS with an appetitive UCS as some orienting response accounts of signaldirected behavior suggest (e.g., Buzsaki, 1982). However, a behavior systems analysis of the interactions of CS, UCS, and motivational state does go some way toward providing a credible model of appetitive Pavlovian performance that predicts not only the instances in which signal-directed behavior either does or does not occur, but the form of that behavior when it is observed.

\section{REFERENCES}

AlberTs, J. R. (1978a). Group behavioral mechanisms of temperature regulation and energy conservation. Journal of Comparative \& Physiological Psychology, 92, 231-243.

Alberts, J. R. (1978b). Huddling by rat pups: Multisensory control of contact. Journal of Comparative \& Physiological Psychology, 92. 220-230

BARNETT, S. A. (1956). Behaviour components in the feeding of wild and laboratory rats. Behaviour, 9, 24-43.

BARNeTt, S. A. (1963). A study in behavior. London: Methuen.

Brown, P. L., \& Jenkins, H. M. (1968). Auto-shaping of the pigeon's key-peck. Joumal of the Experimental Analysis of Behavior, 11, 1-8.

BuzSAKI, G. (1982). The "where is it?" reflex: Autoshaping the orienting response. Journal of the Experimental Analysis of Behovior, 37, $461-484$

CaLhoun, J. B. (1962). The ecology and sociology of the Norway rat. Bethesda, MD: US Dept. of Health, Education and Welfare.

Cleland, G. G., \& DaveY, G. C. L. (1982). The effects of satiation and reinforcer devaluation on signal-centered behavior in the rat. Learning \& Motivation, 13, 343-360.

Cleland, G. G., \& Davey, G. C. L. (1983). Autoshaping in the rat: The effects of localizable visual and auditory signals for food. Journal of the Experimental Analysis of Behavior, 40, 47-56.

DAVEY, G. C. L. (1989). Ecological learning theory. London: Routledge.

Davey, G. C. L., Cleland, G. G. (1982). Topography of signalcentered behavior in the rat: Effects of deprivation state and reinforcer type. Journal of the Experimental Analysis of Behavior, 38, 291-304.

Davey, G. C. L., \& Cleland, G. G. (1984). Food anticipation and lever-directed activities in rats. Learning \& Motivation, 15, 12-36.

Davey, G. C. L., Oakley, D. A., Clelano, G. G. (1981). Autoshaping in the rat: Effects of omission on the form of response. Journal of the Experimental Analysis of Behavior, 36, 75-91.

Davey, G. C. L., Philups, S., Cleland, G. G. (1981). The topography of signal-centred behaviour in the rat: The effects of solid and liquid food reinforcers. Behaviour Analysis Letters, 1, 331-337.

EwER, R. F. (1971). The biology and behavior of a free-living population of black rats (Rattus rattus). Animal Behavior Monographs, 4, 127-174.

GALEF, B. G., JR. (1978). Differences in affiliation behavior of weanling rats selecting eating and drinking sites. Journal of Comparative \& Physiological Psychology, 92, 431-437.

GrastyAN, E., \& VERECZKEI, L. (1974). Effects of spatial separation of the conditioned signal from the reinforcement: A demonstration of the conditioned character of the orienting response or the orientational character of conditioning. Behavioral Biology, 10, 121-146.

GRIFFITHS, S., \& DAVEY, G. C. L. (1984). Signal-directed behaviour in rats using a heat UCS. IRCS Medical Science, 12, 1060-1061.

HARRIson, J. M. (1979). The control of responding by sounds: Unusual effect of reinforcement. Journal of the Experimental Analysis of Behavior, 32, 167-181.

Holland, P. C. (1984). Origins of behavior in Pavlovian conditioning. Psychology of Leaming \& Motivation, 129-174.

Jenkins, H. M., Moore, B. R. (1973). The form of the auto-shaped 
response with food or water reinforcers. Journal of the Experimental Analysis of Behavior, 20, 163-182.

Pavlov, I. P. (1927). Conditioned reflexes. Oxford, England: Oxford University Press.

Peterson, G., Ackil, J. E., Frommer, G. P., \& Hearst, E. S. (1972). Conditioned approach and contact behavior toward signals for food or brain stimulation reinforcement. Science, 177, 1009-1011.

Schwartz, B., \& Gamzu, E. (1977). Pavlovian control of operant behavior. In W. K. Honig \& J. E. R. Staddon (Eds.), Handbook of operant behavior. Engelwood Cliffs, NJ: Prentice-Hall.

STEININGER, F. von. (1950). Beitrage zur Sociologie und sonstigen Biologie der Wanderatte. Zeitschrift für Tierpsychologie, 7, 356-379.

Stimbert, V. E., Schaeffer, R. W., \& Grimsley, D. L. (1966). Acquisition of an imitative response in rats. Psychonomic Science, 5 , 339-340.

TIMBERLAKE, W. (1983). The functional organization of appetitive be- havior: Behavior systems and learning. In M. D. Zeiler \& P. Harzem (Eds.), Advances in analysis of behavior: Vol. 3. Biological factors in learning. Chichester, England: Wiley.

TIMBERLAKE, W., \& GRANT, D. L. (1975). Autoshaping in rats to the presentation of another rat predicting food. Science, 190, 690-692.

Wasserman, E. A. (1973). Pavlovian conditioning with heat reinforcement produces stimulus-directed pecking in chicks. Science, 181, 875-877.

Wasserman, E. A., Hunter, N. B., Gutowski, K. A., Bader, S. A. (1975). Auto-shaping chicks with heat reinforcement: The role of stimulus-reinforcer and response-reinforcer relations. Journal of Experimental Psychology, 104, 158-189.

(Manuscript received September 15, 1988; revision accepted for publication December 14, 1988.)

\section{Announcement}

\section{Psychologists for the Ethical Treatment of Animals Call for Papers}

Psychologists for the Ethical Treatment of Animals (PsyETA) is soliciting manuscripts for Volume 4 of Humane Innovations and Alternatives to Animal Experimentation: A Notebook. We invite short papers describing specific ways to minimize suffering and harm of laboratory animals. Areas of particular interest include husbandry and housing environments, experimental design, experimental procedure, and teaching methods.

Manuscripts are refereed by a distinguished board of editors who include scientists in biomedicine, toxicology and ethology, as well as psychology.

For more information and author guidelines, write to Emmanuel Bernstein, Glenwood Estates, Saranac Lake, NY 12983, or phone 518-891-4140.

(Volume 3 is now available and features over 25 brief articles [76 pp.]. The cost is $\$ 8$ for PsyETA members and $\$ 12$ for nonmembers. Send to PsyETA, P.O. Box 87, New Gloucester, ME 04260.) 